\title{
Conformal Sector in $D=6$ Quantum Gravity
}

\author{
Sergei D. Odintsov 1 and August Romeo, \\ Tomsk Pedagogical Institute, \\ 634041 Tomsk, Russian Federation, \\ and Department E.C.M., Faculty of Physics, University of Barcelona, \\ Diagonal 647, 08028 Barcelona, Spain
}

\begin{abstract}
We discuss the conformal factor dynamics in $D=6$. Accepting the proposal that higher-derivative dimensionless terms in the anomaly-induced effective action may be dropped, we obtain a superrenormalizable (like in $D=4$ ) effective theory for the conformal factor. The one-loop analysis of this theory gives the anomalous scaling dimension for the conformal factor and provides a natural mechanism to solve the cosmological constant problem.
\end{abstract}

1. Introduction. After the discovery of the conformal anomaly [1] (see also [目]) it was found that it plays an important role in different physical situations; among them one can mention string theory [3], the C-theorem and its generalizations [4], local and non-local anomaly-induced action in $D=4$ [5, 6], anomaly structure in $D$ dimensions [7] and some other issues 8 .

In the recent work [6] (see [9]-11], [14] for further development), the effective theory for the conformal factor induced by the conformal anomaly in $D=4$ quantum gravity has been suggested. The model of ref. [6] is supposed to describe the effective theory of quantum gravity in the infrared region (at large distances) before the GUT epoch. Note that the model of ref. [6] was constructed in close analogy with $D=2$ induced quantum gravity [3] which, however,

\footnotetext{
${ }^{1}$ E-mail: odintsov @ ebubecm1.bitnet.
} 
may be realized exactly due to specific properties of the dimension $D=2$.

A very interesting question appears: can a reliable effective theory of quantum gravity based on anomaly-induced dynamics be constructed for increasing (even) dimensions $D>4$ ? That is the purpose of this work: to clarify some issues connected with this question, using $D=6$ as an explicit example. In particular, we discuss the structure of the anomaly-induced action and construct the conformal sector in $D=6$ quantum gravity.

2. D-dimensional theory. First of all, let us describe a general situation in a $D$ dimensional spacetime ( $D$ is even). Starting from conformally invariant free matter in such a space, we may explicitly find the conformal anomaly $T_{A}$ which results from using regularization to remove the infinities [1]. $T_{A}$ consists of $D$-dimensional geometrical invariants which may be divided into two groups: conformally invariant ones and total derivative invariants. In the simplest case, $D=2, T_{A}=c R$, where $R$ is a total derivative and the central charge $c$ consists of the contribution of different spin fields (including the gravitational field itself).

Choosing the simplest conformal parametrization

$$
g_{\mu \nu}=e^{2 \sigma} \eta_{\mu \nu}
$$

where $\sigma$ is the conformal factor and $\eta_{\mu \nu}$ is the flat metric, one can present the conformal anomaly as

$$
T_{A}=\frac{\delta}{\delta \sigma(x)} S_{\text {anom }}
$$

and find the anomaly-induced action $S_{\text {anom }}$ by explicit solution of (2) [3, 5]. Note that only in $D=2$ does parametrization (1) completely fix the gauge, and hence $S_{\text {anom }}$ is unique. In $D \neq 2$ $S_{\text {anom }}$ is defined only up to some conformal invariant.

Integrating eq. (2) in parametrization (1) one can find the general structure of $S_{\text {anom }}$ in the form

$$
S_{\mathrm{anom}}=\int d^{D} x\left\{\tau \sigma \square^{D / 2} \sigma+V\left(\sigma, \nabla_{\mu} \sigma, \square \sigma\right)\right\}
$$

where $\tau$ is some numerical coefficient and $V$ is a complicated interaction potential whose structure is not unique. In particular, for $D=2 V=0$, for $D=4 V=\tau_{1}\left[\square \sigma+\left(\partial_{\mu} \sigma\right)^{2}\right]^{2}$, and so 
on. The coefficient $\tau_{1}$, as well as $\tau$, are defined by by the coefficients of the conformal anomaly (and hence by the number of fields of different spins in the theory).

In order to describe the anomaly-induced dynamics, one has to add to $S_{\text {anom }}$ (3) the classical action of the gravitational field (in parametrization (10)). In $D=2$ this is simply the cosmological constant term, and in $D=4$ the Einstein gravity with cosmological term. Then, in $D=2$ we have the complete theory. However, in $D>2$ we have to argue (as was done in [6]) that the contribution from spin-2 excitations is suppressed and we may consider the effective theory of the conformal factor. This proposal was partially justified in $D=4$ [9] by showing that spin-2 gravitational degrees of freedom, (for a particular model of quantum gravity) in some approximation, only give a finite contribution to the coefficients of the conformal anomaly, and hence may be taken into account afterwards. We will accept that a similar property holds in general $D$. Another proposal which has been made in [6] is that $V$ in (3) may be dropped. As was shown in ref. [6], in $D=4$ this may be justified by the fact that the effective $\sigma$-theory possesses an infrared fixed point, and setting the corresponding coupling constant to zero leads to $V=0$ at this fixed point, where we describe the effective theory for quantum gravity in infrared. We will again accept this proposal in arbitrary $D$. Of course, it is extremely difficult to check in $D=6$, due to the number of higher-derivative dimensionless terms in the effective action (only one such term is present at $D=4$ ).

3. Conformal factor theory. Let us start the construction of the trace anomalyinduced action in the $D=6$ theory. The standard strategy, applied in the $D=4$ theory is to start from the conformal anomaly, integrate over it, and obtain the anomaly-induced action. That is the action (plus Einstein theory) which was used in ref. [6] to describe the IR sector of quantum gravity. Such procedure is not difficult to do in $D=4$, where the conformal anomaly for conformally invariant fields includes only 3 terms.

However, in $D=6$ life is much harder. In particular, let us consider the theory for a 
free conformally invariant scalar field in $D=6$ :

$$
S=\int d^{6} x \sqrt{-g}\left\{-\frac{1}{2} \varphi \square \varphi+\frac{1}{10} R \varphi^{2}\right\}
$$

Then, the conformal anomaly (without total derivative terms like $\square R$ ) is given by

$$
\begin{aligned}
T_{A}=\frac{1}{(4 \pi)^{3}} \int d^{6} x \sqrt{-g} & \left\{-\frac{23}{12 \cdot 9450} R \square R+\frac{13}{22680} R_{\mu \nu} \square R^{\mu \nu}+\frac{1}{6480} R^{\mu \nu \alpha \beta} \square R_{\mu \nu \alpha \beta}\right. \\
& -\frac{1}{6} \frac{1}{27000} R^{3}+\frac{1}{180 \cdot 30} R R_{\mu \nu} R^{\mu \nu}-\frac{1}{180 \cdot 30} R R_{\mu \nu \alpha \beta} R^{\mu \nu \alpha \beta} \\
& -\frac{1}{1260} R_{\mu \nu} R_{\alpha}^{\mu} R^{\nu \alpha}+\frac{1}{2268} R_{\mu \nu} R_{\alpha \beta} R^{\mu \nu \alpha \beta}-\frac{1}{5670} R_{\mu \nu} R^{\mu \lambda \rho \sigma} R_{\lambda \rho \sigma}^{\nu} \\
& \left.+\frac{1}{1890} R_{\mu \nu \rho \sigma} R_{\alpha \beta}^{\mu \nu} R^{\rho \sigma \alpha \beta}\right\}
\end{aligned}
$$

Taking into account all the total derivative terms (by using the corresponding $a_{3}$ coefficient of the Schwinger-De Witt expansion[12], see [13]), the number of terms in (5) increases a lot. The straightforward integration of such an expression over the conformal anomaly is a very hard task.

Here we shall outline an alternative and efficient method to construct the required action, already proposed in [6]. The chief idea is that, according to the observations made in that paper, both scale and conformal invariance must be preserved (taking into account the transformation of the integration measure $d^{D} x$ ). This fixes - up to total divergence - not just the type of terms which can be present, but also their relative coefficients. Let $\varphi=e^{\sigma}$; in order to maintain scale invariance in $D=6$, we must have a combination of (integrated) quotients containing the same number of $\varphi$ 's in the numerator and in the denominator and with six derivatives in the numerator:

$$
\begin{aligned}
& \left(\frac{\partial \varphi}{\varphi}\right)^{2}\left(\frac{\partial \varphi}{\varphi}\right)^{2}\left(\frac{\partial \varphi}{\varphi}\right)^{2} \\
& \left(\frac{\partial \varphi}{\varphi}\right)^{2}\left(\frac{\square \varphi}{\varphi}\right)^{2}, \quad \ldots, \frac{\partial_{\mu} \varphi \partial_{\nu} \varphi \partial_{\lambda} \varphi \partial^{\mu} \partial^{\nu} \partial^{\lambda} \varphi}{\varphi^{4}} \\
& \left(\frac{\square \varphi}{\varphi}\right)^{3}, \quad \ldots, \frac{\partial_{\mu} \varphi \partial_{\nu} \partial_{\lambda} \varphi \partial^{\mu} \partial^{\nu} \partial^{\lambda} \varphi}{\varphi^{3}} \\
& \frac{\partial_{\mu} \square \varphi \partial^{\mu} \square \varphi}{\varphi^{2}}, \quad \ldots, \frac{\partial_{\mu} \varphi \partial^{\mu} \square^{2} \varphi}{\varphi^{2}}, \\
& \frac{\square^{3} \varphi}{\varphi}
\end{aligned}
$$


On the whole, there are 23 of them. Taken as a set, they are not conformally invariant. In fact, the most general Lagrangian will be a sum of all the independent conformally-invariant combinations which can be constructed with these building blocks. We put the infinitesimal expression of a conformal transformantion in the way:

$$
\begin{aligned}
& \delta \varphi(x)=2 \tilde{\alpha} \varepsilon \cdot x \varphi(x) \\
& \delta x^{\mu}=-\varepsilon_{\nu}\left(2 x^{\mu} x^{\nu}-\eta^{\mu \nu} x^{2}\right),
\end{aligned}
$$

where the $\varepsilon_{\mu}$ 's are the infinitesimal parameters and $\tilde{\alpha}=D / 2-1$, as usual.

The quantity $d^{D} x\left(\frac{\square \varphi}{\varphi}\right)^{3}$ is already conformally invariant in $D=6$ and will therefore be one of the pieces in our Lagrangian, as it stands. However, this case is rather execeptional, and in general one has to perform lengthy examinations of the transformed versions for each term so as to construct the desired invariant combinations. We illustrate this with an example: the terms $d^{D} x\left(\frac{\square \varphi}{\varphi}\right)^{2}\left(\frac{\partial \varphi}{\varphi}\right)^{2}, d^{D} x\left(\frac{\partial_{\mu} \partial_{\nu} \varphi}{\varphi}\right)^{2} \frac{\square \varphi}{\varphi}$ are not conformally invariant when taken separately. However, the linear combination $d^{D} x\left[5\left(\frac{\square \varphi}{\varphi}\right)^{2}\left(\frac{\partial \varphi}{\varphi}\right)^{2}-2\left(\frac{\partial_{\mu} \partial_{\nu} \varphi}{\varphi}\right)^{2} \frac{\square \varphi}{\varphi}\right]$ actually is (in $D=6$ ), and may be included in our action. Thus,

$$
S_{\text {anom }}=\int d^{D} x\left\{\tau^{2}\left(\frac{\square \varphi}{\varphi}\right)^{3}+\rho\left[5\left(\frac{\square \varphi}{\varphi}\right)^{2}\left(\frac{\partial \varphi}{\varphi}\right)^{2}-2\left(\frac{\partial_{\mu} \partial_{\nu} \varphi}{\varphi}\right)^{2} \frac{\square \varphi}{\varphi}\right]+\ldots\right\}
$$

We will not write explicitly all terms in (8), as the corresponding expression would take more than one page and is not really used in the explicit analysis below.

After taking this process to the end, one should get an action $S_{\text {ext }}$ giving the anomalyinduced action with contributions from the classical theory, i.e. $S_{\mathrm{ext}}=S_{\mathrm{anom}}+S_{\mathrm{grav}}$. Of course, the connection between the coefficients in such an action and those in the conformal anomaly is absent.

In $S_{\text {ext }}$, the first term $S_{\text {anom }}$ corresponds to the integration of the conformal anomaly, while the second comes from the corresponding ones in the classical action for gravity in $D=6$ :

$$
S_{\text {grav }}=\int d^{6} x \sqrt{-g}\left\{\lambda+\tilde{\gamma}_{1} R\right\}
$$


Thus, our analog of the $4 D$ action in ref. [6] looks like (we drop also derivative interactions in the dimensionless term $S_{\text {anom }}$, supposing that, like in $D=4$, this corresponds to an IR stable point of the theory or there exists some other mechanism for its vanishing.)

$$
\begin{gathered}
S_{\mathrm{ext}}=\int d^{D} x \mathcal{L}[\sigma(x)], \\
\mathcal{L}[\sigma]=\tau^{2} \sigma \square^{3} \sigma+\lambda e^{6 \sigma}+\gamma_{1} e^{4 \sigma}\left[\square \sigma+2(\partial \sigma)^{2}\right] .
\end{gathered}
$$

This expression is taken to be written in Euclidean space. Then, (we adopt new notations from this point), changing $\sigma$ as

$$
\sigma \rightarrow \alpha \sigma
$$

and - after dividing by $\alpha^{2}$ - the Lagrangian for the conformal-anomaly induced dynamics can be put into the form

$$
\mathcal{L}[\sigma]=\tau^{2} \sigma \square^{3} \sigma+\frac{\lambda}{\alpha^{2}} e^{6 \alpha \sigma}-2 \gamma_{1} e^{4 \alpha \sigma}(\partial \sigma)^{2} .
$$

That will be our starting point. As we will see, this theory in $D=6$ is superrenormalizable, as in $D=4$.

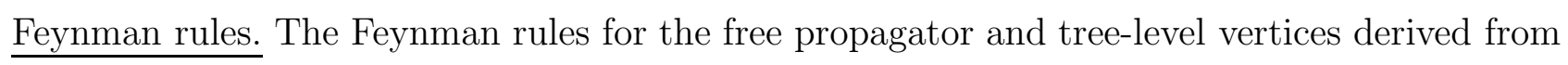
this action have the following expression

Free propagator

$$
-\frac{1}{2} \frac{1}{\tau^{2} k^{6}+2 \gamma_{1} k^{2}-18 \lambda}
$$

$\lambda$-vertex

$$
-\frac{\lambda}{\alpha}(6 \alpha)^{n}
$$

$\gamma_{1}$-vertex

$$
-\frac{\gamma_{1}}{4 \alpha^{2}}(4 \alpha)^{n} \sum_{\substack{j, l \\ 1 \leq j<l \leq n}}\left(p_{j} \cdot p_{l}\right)
$$




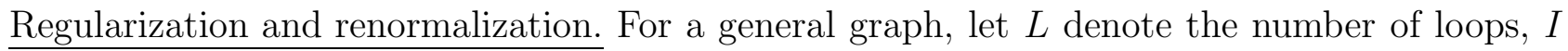
the number of internal propagators, and $V_{\gamma_{1}}$ the number of $\gamma_{1}$-vertices. Combining elementary power counting and the usual topological relation, we derive the value of the superficial degree of divergence $\mathcal{D}$ :

$$
\left.\begin{array}{c}
\mathcal{D}=6 L-6 I+2 V_{\gamma_{1}} \\
L=I-V+1, \quad V \equiv V_{\lambda}+V_{\gamma_{1}}
\end{array}\right\} \Longrightarrow \mathcal{D}=6-6 V_{\lambda}-4 V_{\gamma_{1}} .
$$

Thus, there are only two possible classes of superficially divergent diagrams, which are typified by

$$
\begin{array}{lll}
V_{\lambda}=1 & V_{\gamma_{1}}=0 & \mathcal{D}=0, \\
V_{\lambda}=0 & V_{\gamma_{1}}=1 & \mathcal{D}=2 .
\end{array}
$$

Note that, as a result of working in $D=6$, this theory is even more convergent that the analogous one developed for $D=4$ in [6].

For $L=1$ (one-loop), the calculation of any of these diagrams involves - at least for the simplest combinations of vanishing external momenta - integrals of the generic sort

$$
\mathcal{I}_{I}^{p} \equiv \int \frac{d^{D} k}{(2 \pi)^{D}} \frac{k^{2 p}}{\left(k^{6}-a k^{4}-b k^{2}-c\right)^{I}} .
$$

By dimensional regularization in

$$
D=6-2 \varepsilon
$$

dimensions, we have been able to calculate the following cases

$$
\begin{aligned}
& \mathcal{I}_{I}^{3 I-3}=\frac{1}{(4 \pi)^{3}} \frac{1}{2 \varepsilon}+O\left(\varepsilon^{0}\right), \\
& \mathcal{I}_{I}^{3 I-2}=\frac{1}{(4 \pi)^{3}} \frac{I a}{2 \varepsilon}+O\left(\varepsilon^{0}\right), \\
& \mathcal{I}_{I}^{3 I-1}=\frac{1}{(4 \pi)^{3}} \frac{I\left[b+(I+1) \frac{a^{2}}{2}\right]}{2 \varepsilon}+O\left(\varepsilon^{0}\right) .
\end{aligned}
$$

Since in our theory we have no $k^{4}$ term, the second integral is actually finite. From these, it is also possible to obtain integrals like

$$
\mathcal{I}_{1}^{\mu \nu} \equiv \int \frac{d^{D} k}{(2 \pi)^{D}} \frac{k^{\mu} k^{\nu}}{k^{6}-a k^{4}-b k^{2}-c}=\frac{1}{D} \mathcal{I}_{1}^{1} \delta^{\mu \nu} .
$$


All of them will be necessary for the evaluation of the one-loop graphs to be studied. $\underline{\text { Results for some one-loop diagrams }}$

- $L=1, V_{\lambda}=1, V_{\gamma_{1}}=0$, with $n$ external legs carrying no momentum:

$$
\frac{\lambda}{2 \alpha^{2} \tau^{2}}(6 \alpha)^{n+2} \frac{1}{(4 \pi)^{3}} \frac{1}{2 \varepsilon}+O\left(\varepsilon^{0}\right)
$$

- $\quad-L=1, V_{\lambda}=0, V_{\gamma_{1}}=1$, with $n$ external legs carrying no momentum (derivatives acting on the loop line only): in this situation, the diagram turns out to be finite.

- $L=1, V_{\lambda}=0, V_{\gamma_{1}}=1$, with $n+2$ external legs, two of them carrying momenta $p$ and $-p$, and the remainig $n$ carrying no momentum (derivatives acting on these external lines only):

$$
-\frac{\gamma_{1}}{8 \alpha^{2} \tau^{2}}(4 \alpha)^{n+4} \frac{1}{(4 \pi)^{3}} p^{2} \frac{1}{2 \varepsilon}+O\left(\varepsilon^{0}\right)
$$

Beta functions. By selecting particular cases of the above diagrams, we renormalize the twopoint function. After taking into account the classical scaling contributions like in [6], we find the one-loop beta functions for the $\lambda$ and $\gamma_{1}$ couplings to be

$$
\begin{aligned}
& \beta_{\lambda}=(6-6 \alpha) \lambda_{r}+9 \frac{\alpha^{2}}{(4 \pi)^{3}} \frac{\lambda_{r}}{\tau^{2}}+\ldots \\
& \beta_{\gamma_{1}}=(4-4 \alpha) \gamma_{1 r} \quad-8 \frac{\alpha^{2}}{(4 \pi)^{3}} \frac{\gamma_{1 r}}{\tau^{2}}+\ldots
\end{aligned}
$$

Similarly, one can find the two-loop, etc corrections. We stop at the one-loop stage. The solutions of $\beta_{\gamma_{1}}=0$ for $\gamma_{1 r} \neq 0$ are $\alpha=\alpha_{ \pm} \equiv \frac{-1 \pm \sqrt{1+8 /\left((4 \pi)^{3} \tau^{2}\right)}}{4 /\left((4 \pi)^{3} \tau^{2}\right)}$, This result is analogous to the anomalous scaling dimension in $D=2$ [16] or in $D=4$ [6]. Note that, in the above relation, $\tau^{2}$ plays the role of the central charge in $D=6$ and $\tau_{c r}^{2}=-8(4 \pi)^{-3}$ corresponds to the $c$-barrier in $D=6$. 
As happens in $D=4$, the classical scaling dimension is obtained from the positive branch of $\alpha_{ \pm}$in the limit of no QG $\left(\tau^{2} \rightarrow \infty\right)$.

$$
\alpha=\alpha_{+} \equiv \frac{-1+\sqrt{1+8 /\left((4 \pi)^{3} \tau^{2}\right)}}{4 /\left((4 \pi)^{3} \tau^{2}\right)},
$$

which is associated to the physical solution.

Now, we turn to the analysis of the first relation in (24). In $D=4$ dimensions, the analog of this equation has led to fixing the cosmological constant in terms of the Newton constant [6]. Now, in $D=6$ this is no longer the case. Instead, after substituting the value of $\alpha_{ \pm}$into (24), we get the relation

$$
\beta_{\lambda}=-\frac{3 \alpha^{2}}{(4 \pi)^{3}} \frac{\lambda_{r}}{\tau^{2}} .
$$

As one can see, it is not possible to make $\beta_{\lambda}$ vanish unless $\lambda_{r}=0$. Hence, one may naturally obtain the solution of the cosmological constant problem in $D=6$.

Note that, in principle, the anomalous scaling dimension could be determined from the first expression in (24). Then, it would give a different value for $\alpha$, making the gravitational coupling constant vanish. Eventually, that case should correspond to the unphysical region of QG, and we do not discuss it.

Summing up, we have constructed the conformal sector of $6 D$ quantum gravity and calculated the anomalous scaling dimension of the conformal factor. As a result, we have got a very natural solution of the cosmological constant problem in $D=6$. Of course, there are still many questions left to be understood in the future. In particular, our proposal to drop higher-derivative dimensionless terms — whose number is quite large - as was done in $D=4$ (where it happens to correspond to a stable IR fixed point) should be examined in more detail. Note that this amounts to extremely lengthy calculations.

Another remark is connected with the fact that in $D=6$, unlike in $D=4$, one can also add four-derivative terms to the classical gravitational action. For instance, if we add a $\gamma_{2} R^{2}$ term to (9), the above one-loop analysis would be completely changed with the result (instead 
of $(24))$ :

$$
\begin{aligned}
& \beta_{\lambda}=(6-6 \alpha) \lambda_{r}+\frac{\alpha^{2}}{(4 \pi)^{3}}\left[9 \frac{\lambda_{r}}{\tau^{2}}-\frac{1}{6} \frac{\gamma_{1 r} \gamma_{2 r}}{\tau^{4}}+\frac{3}{72} \frac{\gamma_{2 r}^{3}}{\tau^{6}}\right]+\ldots, \\
& \beta_{\gamma_{1}}=(4-4 \alpha) \gamma_{1 r}+\frac{\alpha^{2}}{(4 \pi)^{3}}\left[-8 \frac{\gamma_{1 r}}{\tau^{2}}+\frac{61}{64} \frac{\gamma_{2 r}^{2}}{\tau^{4}}\right]+\ldots \\
& \beta_{\gamma_{2}}=(2-2 \alpha) \gamma_{2 r}+\frac{\alpha^{2}}{(4 \pi)^{3}} \frac{1}{2} \frac{\gamma_{2 r}}{\tau^{2}}+\ldots
\end{aligned}
$$

The analysis of these equations is more similar to the case of ref. [6].

Our main conclusion is that the principal possibility of realizing the conformal factor dynamics in $D>4$ still exists. However, the explicit details of such a realization are much more complicated.

We would like to thank Ignatios Antoniadis for very stimulating discussions and participartion at the early stages of this work.

\section{References}

[1] S. Deser, M. Duff and C.J. Isham, Nucl. Phys. B111 (1976) 45.

[2] L.S. Brown and J.P. Cassidy, Phys. Rev. D 15 (1977) 2810; for a review see N. Birrell and P. Davies, Quantum Fields in Curved Space-time, C.U.P. 1982, and A.A. Grib, S.G. Mamaev and V.M. Mostepanenko, Quantum Effects in Intensive External Fields, Atomizdat, Moscow, 1980.

[3] A.M. Polyakov, Phys. Lett. B103 (1981) 207.

[4] A.B. Zamolodochikov, JETP Lett. 43 (1986) 730;

J.L. Cardy, Phys. Lett. B215 (1988) 749;

I. Jack and H. Osborn, Nucl. Phys. B343 (1990) 647;

A. Capelli, D. Friedan and J.I. Latorre, Nucl. Phys. B352 (1991) 616;

A. Capelli, J.I. Latorre and X. Vilasís-Cardona, Nucl. Phys. B 376 (1992) 510. 
[5] R. Reigert, Phys. Lett. B134 (1984) 56;

I.L. Buchbinder, S.D. Odintsov and I.L. Shapiro, Phys. Lett. 162 (1985) 93;

E.S. Fradkin and A.A. Tseytlin, Phys. Lett. B134 (1984) 187;

E.T. Tomboulis, Nucl. Phys. B329 (1990) 410;

S.D. Odintsov and I.L. Shapiro, Class. Quantum Grav. 8 (1991) L57;

J.S. Dowker, preprint MUTP-14 (1994).

[6] I. Antoniadis and E. Mottola, Phys. Rev. D 46 (1992) 2013.

[7] S. Deser and A. Schwimmer, Phys. Lett. B309 (1993) 279;

H. Osborn and A. Petkos, preprint DAMTP/93-31 (1993).

[8] F. Bastianelli and P. van Nieuwenhuizen, Nucl. Phys. B389 (1993) 53.

[9] I. Antoniadis, P.O. Mazur and E. Mottola, Nucl. Phys. B388 (1992) 627;

S.D. Odintsov, Z. Phys. C54 (1992) 531.

[10] I. Antoniadis and S.D. Odintsov, Mod. Phys. Lett. A8 (1993) 979.

[11] E. Elizalde, S.D. Odintsov and I.L. Shapiro, Barcelona preprint UB-ECM-PF 94/11 (1994); Class. Quantum Grav., to appear.

[12] B.S. De Witt, Dynamical Theory of Groups and Fields, Gordon and Breach, N.Y., 1965.

[13] P.B. Gilkey, J. Diff. Geom. 10 (1975) 601.

[14] S.D. Odintsov and R. Percacci, preprint HEPTH 9404020; Mod. Phys. Lett. A, to appear.

[15] I.L. Buchbinder, S.D. Odintsov and I.L. Shapiro, Effective Action in Quantum Gravity, IOP Publishing, Bristol and Philadephia, 1992).

[16] A.M. Polyakov, V.G. Knizhnik and A.B. Zamolodchikov, Mod. Phys. Lett. A3 (1988) 819 ; 
F. David, Mod. Phys. Lett. A3 (1988) 1651;

J. Distler and H. Kawai, Nucl. Phys. 321 (1989) 509. 